\title{
Privatização do Sistema Único de Saúde (SUS): Uma revisão da literatura a partir de artigos científicos da base de dados da Scielo
}

\author{
Privatization of the Brazilian National Health Service (SUS): A literature review based on scientific \\ articles from the Scielo database \\ Privatización del Sistema Único de Salud (SUS): Una revisión de la literatura basada en artículos \\ científicos de la base de datos Scielo
}

Recebido: 05/04/2021 | Revisado: 12/04/2021 | Aceito: 15/04/2021 | Publicado: 25/04/2021

Bruno Ferreira de Oliveira
ORCID: https://orcid.org/0000-0003-2727-774X
Universidade Cândido Mendes, Brasil
E-mail: brunooliver17@ @otmail.com
Felipe Ponciano da Cruz
ORCID: https://orcid.org/0000-0002-1794-5946
Universidade do Estado do Rio de Janeiro, Brasil
E-mail: flponciano@ gmail.com
Igor Masullo Soares
ORCID: https://orcid.org/0000-0002-9213-0210
Universidade Federal do Rio de Janeiro, Brasil
E-mail: igormsoares@ globo.com
Hélida Ribeiro Hermes de Oliveira
ORCID: https://orcid.org/0000-0003-2409-3363
Universidade Federal Fluminense, Brasil
E-mail: helidaribei@ @otmail.com

\section{Resumo}

O objetivo do artigo é a realização da revisão bibliográfica sobre os estudos relacionados com a privatização do Sistema Único de Saúde (SUS) no Brasil, organizando o estado-da-arte sobre o referido tema e indicando as possíveis lacunas existentes referentes ao assunto, utilizando a base da Scielo entre os anos de 1998 e 2020. Utilizou-se também métricas bibliométricas e Análise de Redes Sociais para subsidiar a discussão dos resultados. Os resultados evidenciam que parte expressiva dos artigos foram publicados entre 2011 e 2020. A FIOCRUZ é a principal instituição associada aos autores da amostra. As principais revistas científicas em termos de quantidade de artigos publicados e em relação ao h5 index são Cadernos de Saúde Pública e Ciência \& Saúde Coletiva, respectivamente. Observa-se entre as revistas científicas a relevância da área de saúde coletiva para os estudos sobre a privatização do SUS e, de forma minoritária, as contribuições da economia, serviço social e da psicologia para o desenvolvimento do tema. A rede de palavras-chave mostra os termos com maiores ocorrências dentre os artigos da amostra. Quanto à rede entre os artigos e os grupos de análise dos elementos privatizantes do SUS, identificou-se que os grupos de análise gestão, financiamento e marco político-institucional, são os mais estudados e os grupos relacionados com a formação profissional e relações de trabalho, participação social e análise comparada são pouco abordados pelos artigos da amostra, representando assim, um caminho interessante para o desenvolvimento de novos estudos relacionados com os elementos privatizantes do SUS.

Palavras-chave: Privatização; Neoliberalismo; SUS; Bibliometria; Análise de redes sociais; Brasil.

\begin{abstract}
The objective of article is to conduct a bibliographic review of studies related to the privatization of the National Health Service (SUS) in Brazil, organizing the state-of-the-art on the topic and indicating possible existing gaps on the subject, using Scielo database, between the years 1998 and 2020. Bibliometric metrics and Social Network Analysis were also used to support the discussion of results. The results show that a significant part of articles was published between 2011 and 2020. FIOCRUZ is the main institution associated with the authors of the sample. The main scientific journals in terms of number of published articles and in relation to the h5 index are Cadernos de Saúde Pública and Ciência \& Saúde Coletiva, respectively. It is observed among the scientific journals the relevance of the collective health area for studies on privatization of the SUS and, as a minority, the contributions of economics, social work and psychology to the development of the theme. The keywords network shows the terms with the highest occurrences among the articles in the sample. As for the network between the articles and the analysis groups of the privatizing elements of the SUS, it was identified that the management, financing and political-institutional framework analysis groups are the most studied, and the groups related to professional training and labor relations,
\end{abstract}


social participation and comparative analysis are little addressed by the articles of the sample, thus representing an interesting path for the development of new studies related to the privatizing elements of the SUS.

Keywords: Privatization; Neoliberalism; SUS; Bibliometrics; Social network analysis; Brazil.

\section{Resumen}

El objetivo del artículo es realizar una revisión bibliográfica sobre los estudios relacionados con la privatización del Sistema Único de Salud (SUS) en Brasil, organizando el estado del arte sobre el tema e indicando las posibles lagunas existentes sobre el mismo, utilizando la base de datos Scielo, entre los años 1998 y 2020. También se utilizaron métricas bibliométricas y análisis de redes sociales para apoyar la discusión de los resultados. Los resultados muestran que una parte importante de los artículos se publicaron entre 2011 y 2020. FIOCRUZ es la principal institución asociada a los autores de la muestra. Las principales revistas científicas en cuanto a número de artículos publicados y en relación con el índice h5 son Cadernos de Saúde Pública y Ciência \& Saúde Coletiva, respectivamente. Se observa entre las revistas científicas la relevancia del área de la salud colectiva para los estudios sobre la privatización del SUS y, de forma minoritaria, las aportaciones de la economía, el servicio social y la psicología al desarrollo del tema. La red de palabras clave muestra los términos con mayor presencia en los artículos de la muestra. En cuanto a la red entre los artículos y los grupos de análisis de los elementos privatizadores del SUS, se identificó que los grupos de análisis gestión, financiación y marco político-institucional son los más estudiados y los grupos relacionados con la formación profesional y las relaciones laborales, la participación social y el análisis comparativo son poco abordados por los artículos de la muestra, representando así un camino interesante para el desarrollo de nuevos estudios relacionados con los elementos privatizadores del SUS.

Palabras clave: Privatización; Neoliberalismo; SUS; Bibliometría; Análisis de redes sociales; Brasil.

\section{Introdução}

A Constituição Federal de 1988 criou o Sistema Único de Saúde (SUS) sob os princípios básicos da Universalidade, Integralidade, Equidade, Descentralização e Participação Social. Não limitado apenas ao atendimento em hospitais e postos de saúde, esse sistema teve em sua concepção a filosofia de prevenção e planejamento da saúde pública, englobando também outras atividades relacionadas, como vacinação e vigilância sanitária.

Assim, o SUS traduz o projeto da reforma sanitária brasileira, colocando o direito à saúde como integrante da construção da cidadania. Essa reforma "indicava um conjunto de mudanças no Estado, na sociedade e na cultura, visando à melhoria da situação de saúde e das condições de vida da população" (Paim \& Teixeira, 2007, p. 1820). Ao estabelecer na Constituição Federal no art. 196 que a saúde é um direito de todos e um dever do Estado, o SUS passa a ser um instrumento importante para o combate à pobreza e a desigualdade social no país (Ocké-Reis, 2012). O SUS possui um caráter “democrático e descentralizado, com responsabilidades estabelecidas para as três esferas de governo" (Paim \& Teixeira, 2007, p. 1820).

Apesar de ser um dos maiores sistemas de saúde público e gratuito do mundo, existem diversos problemas e desafios ao seu pleno desenvolvimento e eficiência, sendo tais empecilhos das mais variadas origens, envolvendo desde questões sobre financiamento e gestão de recursos a dificuldades geradas devido às dimensões físicas e demográficas do Brasil, como o atendimento a regiões de mais difícil acesso.

De forma simultânea ao nascimento do SUS, emergiram tanto nas economias centrais quanto nas periféricas sobretudo na América Latina - os preceitos do neoliberalismo, com a redefinição da relação do Estado na sociedade. Pode-se definir o neoliberalismo como uma ideologia que considera que o bem-estar da sociedade é maximizado pelo individualismo e trata da impossibilidade de intervenção racional na economia, sendo caracterizado pela garantia da liberdade individual, livre iniciativa, mercados desregulados e livre comércio (Harvey, 2007; Ibarra, 2011).

O neoliberalismo surge em meados da década de 1940 como uma "reinvenção" do liberalismo vigente até a crise de 1929 (Anderson, 1995), além de ser uma reação às políticas keynesianas após a grande depressão e também ao consenso construído em torno do Estado de Bem-Estar Social no período do pós-guerra, incluindo a construção de sistemas públicos de saúde, como, por exemplo, o sistema inglês, conhecido como National Health Service (NHS) (Meireles \& El -Aouar, 2010; Kingfisher \& Maskovsky, 2008). 
No período pós-guerra até meados da década de 1970, essa teoria ficou em segundo plano, em virtude do período de prosperidade econômica, aliado à capacidade de planejamento do Estado no crescimento e do desenvolvimento econômico e social. A crise no final da década de 1970 (choques dos preços de Petróleo, aumento do nível de preços e desemprego) foi a oportunidade encontrada para colocar as teorias neoliberais em evidência e prática, principalmente com os governos de Ronald Reagan e Margaret Thatcher.

Assim, pode-se conceituar como políticas econômicas e sociais lastreadas pela ideologia neoliberal

“(...) the privatization of public services, the elimination of subsidies and the restructuring of welfare provisioning to increase attachment to the workforce, and the reform of urban fiscal policies to encourage gentrification and securitization of elite residential and commercial areas" (Kingfisher \& Maskovsky, 2008, p.116).

Nesse contexto, os países latino-americanos, afundados em uma crise fiscal, "adotam" os preceitos neoliberais sistematizados por Oliver Williamson, conhecidos como o Consenso de Washington, ocasionando um enfraquecimento das bases de construção de um estado de bem-estar social na América Latina, sendo a política econômica baseada em austeridade fiscal e com a política social sendo colocada em segundo plano. O tripé neoliberal - desregulamentação, privatização e abertura dos mercados - evidencia a sua relação com a globalização, além de expor uma questão que é muito cara à implementação do desenvolvimento, que é a perda da soberania nacional relacionada à capacidade de implementar políticas de desenvolvimento (Paiola, 2014).

Nesse contexto, ganha cada vez mais espaço dentro do debate público a contestação ao modelo de gestão do SUS, sendo recorrentes propostas de reforma, como a maior participação de entidades privadas dentro do sistema. As mudanças ocorridas no cenário político-institucional durante os anos 90 e 2000 trouxeram consigo o crescimento da presença de atores privados dentro do SUS, sendo tal processo intensificado com a proliferação de modelos privados de gestão, como as Parcerias Público-Privadas (PPPs) e as Organizações Sociais (OSs).

Alinhado a essas transformações vividas no âmbito político-institucional, a discussão cientifica entorno do tema, iniciada ao final dos anos 90, também foi intensificada durante a década de 2010, com um forte aumento das publicações sobre o assunto principalmente a partir de 2016. Dessa forma, mostra-se produtivo para a evolução dos estudos na área a definição de um quadro teórico sobre a privatização do SUS, possibilitando a identificação de gargalos dentro das pesquisas, pontos de maior concordância e áreas que não estão recebendo a devida atenção.

Visando contribuir para a sistematização desse quadro teórico, esse artigo se propõe a realizar uma revisão bibliométrica da literatura nacional acerca dos elementos privatizantes do SUS, a partir da base de dados da Scielo, utilizando métricas bibliométricas e a Análise de Redes Sociais para a organização dos artigos científicos produzidos, sob diferentes matizes.

De uma perspectiva metodológica, buscou-se copilar as publicações mais relevantes sobre a privatização dos serviços de saúde no Brasil e analisar quais elementos se destacam em cada uma delas, visando a aglutinação em grupos que permitam identificar os assuntos mais recorrentemente abordados pelos autores, bem como a análise cartográfica dos artigos, mapeando seus autores, instituições relacionadas e em quais revistas seus artigos foram publicados. Por fim, uma análise de redes sociais utilizando como referência as palavras-chave dos artigos também foi realizada a fim de contribuir para a melhor definição do quadro teórico sobre esse tema e identificar os principais elementos dentro do debate.

Após essa breve sessão introdutória, esse artigo segue para a apresentação da metodologia utilizada na realização da revisão bibliográfica, seguido pela apresentação e análise dos resultados e, por fim, as considerações finais sobre o estudo elaborado. 


\section{Metodologia}

Em consonância com o objetivo do trabalho, a metodologia adotada foi o estudo bibliométrico dos artigos científicos na base de dados da Scientific Electronic Library Online (Scielo) relacionados com a privatização do SUS.

A bibliometria apresenta uma variedade de indicadores relacionados com a atividade e o desenvolvimento científico, objetivando a sistematização da literatura especializada relacionada com a temática apresentada (Chain et al., 2019). Sua utilidade repousa na capacidade de ordenamento e de organização do escopo teórico em discussão, apresentando à comunidade científica o conhecimento estabelecido, bem como os possíveis caminhos futuros de discussão (Do Prado et al, 2016). Os estudos bibliométricos utilizam métricas relacionadas com citações, autorias, palavras-chave, periódicos científicos, base científica e intelectual dos autores da amostra, entre outros (Do Prado et al, 2016; Chain et al., 2019).

O levantamento bibliográfico realizado na base de dados da Scielo relacionadas com os estudos sobre privatização do SUS, foi realizada sem restrição temporal, utilizando os seguintes termos relacionados com a temática: saúde, privatização, SUS, Brasil, neoliberalismo, e saúde pública. Cabe salientar que a única restrição imposta no levantamento foi a busca de artigos citáveis. Abaixo, é apresentado o Quadro 1 que sintetiza o protocolo de busca dos artigos na Scielo realizado nos dias 26 e 27 de fevereiro de 2021.

Quadro 1: Protocolo de busca dos artigos da base de dados da Scielo.

\begin{tabular}{|l|c|}
\hline \multicolumn{1}{|c|}{ Descrição do protoco de busca } & $\begin{array}{c}\text { Quantidade de artigos } \\
\text { encontrados }\end{array}$ \\
\hline $\begin{array}{l}\text { Protocolo 1: (saúde) AND (privatização) AND (sus) AND (brasil) AND } \\
\text { network:org AND -network:rve AND ( is_citable:("is_true") AND type:*) }\end{array}$ & 51 \\
\hline $\begin{array}{l}\text { Protocolo 2: (neoliberalismo) AND (privatização) AND (saúde) AND } \\
\text { network:org AND -network:rve AND ( is_citable:("is_true") AND type:*) }\end{array}$ & \multirow{2}{*}{25} \\
\hline $\begin{array}{l}\text { Protocolo 3: (saúde pública) AND (brasil) AND (privatização) AND } \\
\text { network:org AND -network:rve AND ( is_citable:("is_true") AND type:*) }\end{array}$ & 25 \\
\hline $\begin{array}{l}\text { Protocolo 4: (saúde pública) AND (brasil) AND (neoliberalismo) AND } \\
\text { network:org AND -network:rve AND ( is_citable:("is_true") AND type:*) }\end{array}$ & \multirow{2}{|}{10} \\
\hline
\end{tabular}

Fonte: Autores.

A partir dos resultados dos protocolos de busca apresentados no Quadro 1, a Figura 1 abaixo apresenta o fluxograma de análise de inclusão e exclusão de artigos encontrados. No total, 94 artigos foram selecionados na referida base de dados. As exclusões dos artigos selecionados, que totalizam 67, se devem a artigos em duplicidade e que não guardam relação com a discussão sobre a privatização do SUS. Assim, a amostra final de artigos científicos utilizada abrange 27 artigos. 
Figura 1: Fluxograma de Resultado dos protocolos de busca na base de dados da Scielo e mecanismos de filtragem e seleção dos artigos da amostra.

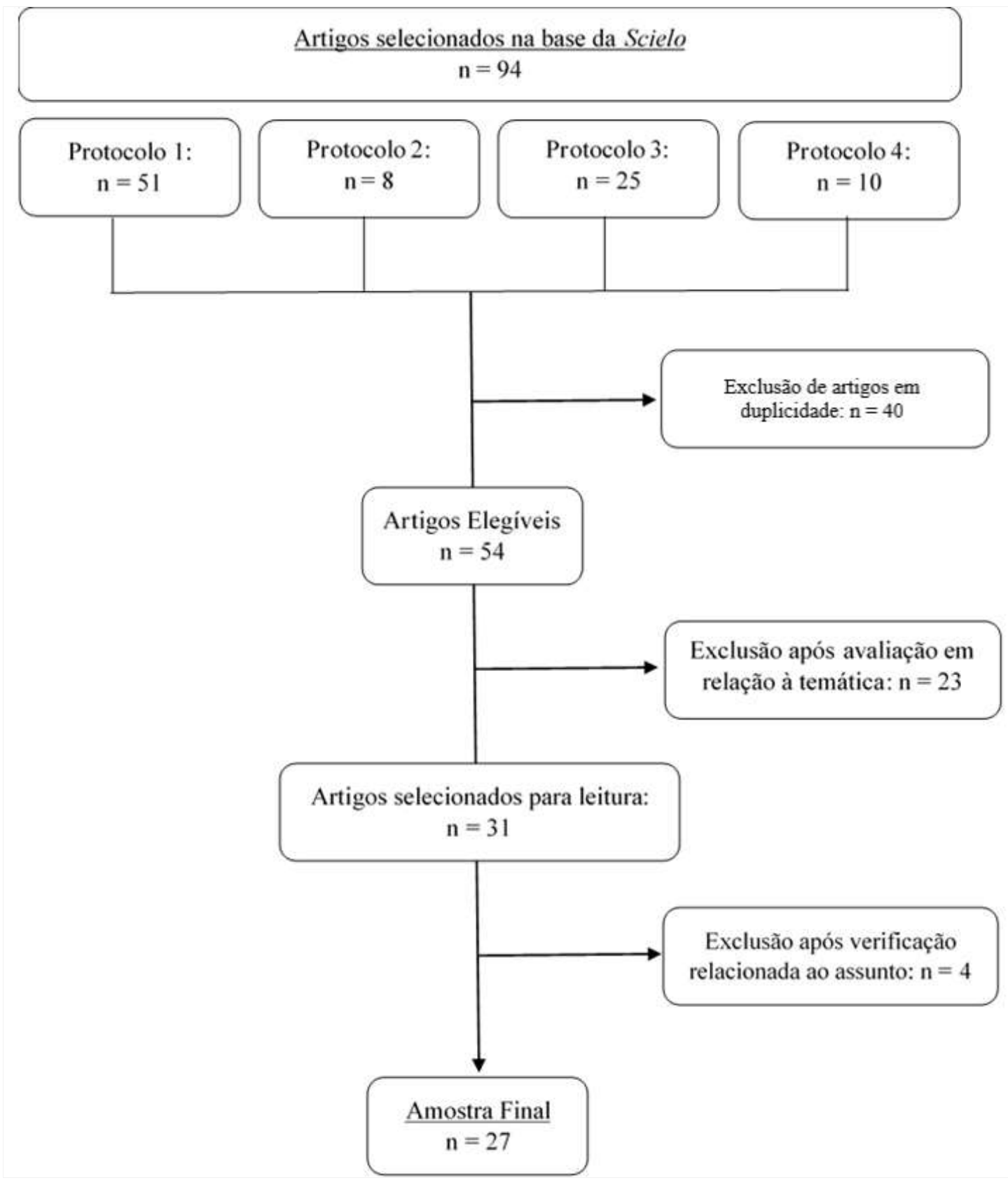

Fonte: Autores.

Assim, apresenta-se no Quadro 2 o detalhamento da amostra final, evidenciando o título do artigo, os autores, o ano de publicação e a revista científica no qual o referido artigo foi publicado. Desta forma, para o estudo da bibliografia que trata da privatização do SUS, utilizou-se as métricas de análise temporal dos artigos da amostra, das principais revistas e suas respectivas área de concentração, das principais instituições relacionadas com estudo do tema, bem como as principais palavras-chave dos artigos da amostra (Do Prado et al, 2016; Chain et al., 2019). 
Quadro 2: Lista dos artigos da amostra, por título, auto, ano e revista científica.

\begin{tabular}{|c|c|c|c|}
\hline$\#$ & $\begin{array}{c}\text { TÍTULO DO ARTIGO } \\
\end{array}$ & AUTOR E ANO & RESVISTA CIENTÍFICA \\
\hline 1 & $\begin{array}{l}\text { Sobre participação popular: uma questão de } \\
\text { perspectiva }\end{array}$ & Valla (1998) & Cadernos de Saúde Pública \\
\hline 2 & Profae e lógica neoliberal: estreitas relações & $\begin{array}{l}\text { Cêa, Reis e Conterno } \\
(2007)\end{array}$ & Trabalho, Educação e Saúde \\
\hline 3 & $\begin{array}{l}\text { O regime regulatório e o mercado de planos } \\
\text { de saúde no Brasil }\end{array}$ & Costa (2008) & Ciência \& Saúde Coletiva \\
\hline 4 & $\begin{array}{l}\text { Formação em fisioterapia no Brasil: reflexões } \\
\text { sobre a expansão do ensino e os modelos de } \\
\text { formação }\end{array}$ & Bispo Júnior (2009) & $\begin{array}{l}\text { História, Ciências, Saúde- } \\
\text { Manguinhos }\end{array}$ \\
\hline 5 & $\begin{array}{l}\text { Globalização e desafios urbanos: Políticas } \\
\text { públicas e desigualdade social nas cidades } \\
\text { brasileiras }\end{array}$ & Pimenta e Pimenta (2011) & EURE (Santiago) \\
\hline 6 & $\begin{array}{l}\text { Lutas sociais pela saúde pública no Brasil } \\
\text { frente aos desafios contemporâneos }\end{array}$ & Santos (2013) & Revista Katálysis \\
\hline 7 & $\begin{array}{l}\text { Comunidade epistêmica e a formação da } \\
\text { reforma sanitária no Brasil }\end{array}$ & Costa (2014) & $\begin{array}{l}\text { Physis: Revista de Saúde } \\
\text { Coletiva }\end{array}$ \\
\hline 8 & $\begin{array}{l}\text { Universalidade da saúde no Brasil e as } \\
\text { contradições da sua negação como direito de } \\
\text { todos }\end{array}$ & Sousa (2014) & Revista Katálysis \\
\hline 9 & $\begin{array}{l}\text { Mudanças no capitalismo contemporâneo e } \\
\text { seu impacto sobre as políticas estatais: o SUS } \\
\text { em debate }\end{array}$ & $\begin{array}{l}\text { Campos, Viana e Soares } \\
\text { (2015) }\end{array}$ & Saúde e Sociedade \\
\hline 10 & $\begin{array}{l}\text { Privatização da gestão do sistema municipal } \\
\text { de saúde por meio de Organizações Sociais na } \\
\text { cidade de São Paulo, Brasil: caracterização e } \\
\text { análise da regulação }\end{array}$ & Contreiras e Matta (2015) & Cadernos de Saúde Pública \\
\hline 11 & $\begin{array}{l}\text { A Terceirização na saúde pública: formas } \\
\text { diversas de precarização do trabalho }\end{array}$ & Druck (2016) & Trabalho, Educação e Saúde \\
\hline 12 & $\begin{array}{l}\text { Benefícios para alguns, prejuízos para muitos: } \\
\text { razões e implicações da adoção da dupla porta } \\
\text { de entrada em hospitais universitários }\end{array}$ & Silva e Caxias (2016) & Saúde e Sociedade \\
\hline 13 & $\begin{array}{l}\text { Reforma sanitária e e privatização da saúde } \\
\text { em um contexto biopolítico de garantia de } \\
\text { direitos }\end{array}$ & $\begin{array}{l}\text { Lara, Guareschi e } \\
\text { Bernardes (2016) }\end{array}$ & Psicologia \& Sociedade \\
\hline 14 & $\begin{array}{l}\text { Vivências de trabalhadores da saúde em face } \\
\text { da lógica neoliberal: um estudo da atenção } \\
\text { básica na Colômbia e no Brasil }\end{array}$ & $\begin{array}{l}\text { Garrido-Pinzón e } \\
\text { Bernardo (2017) }\end{array}$ & Cadernos de Saúde Pública \\
\hline
\end{tabular}

\begin{tabular}{|c|c|c|c|}
\hline \# & $\begin{array}{l}\text { TÍTULO DO ARTIGO } \\
\end{array}$ & AUTOR E ANO & RESVISTA CIENTÍFICA \\
\hline 15 & $\begin{array}{l}\text { Descaminhos da seguridade social e } \\
\text { desproteção social no Brasil }\end{array}$ & Sposati (2018) & Ciência \& Saúde Coletiva \\
\hline 16 & $\begin{array}{l}\text { Governos Lula e Dilma em matéria de } \\
\text { seguridade social e acesso à educação superior }\end{array}$ & $\begin{array}{l}\text { Marques, Ximenes e } \\
\text { Ugino (2018) }\end{array}$ & $\begin{array}{l}\text { Brazilian Journal of } \\
\text { Political Economy }\end{array}$ \\
\hline 17 & $\begin{array}{l}\text { Notas sobre o futuro do SUS: breve exame de } \\
\text { caminhos e descaminhos trilhados em um } \\
\text { horizonte de incertezas e desalentos }\end{array}$ & Noronha et al. (2018) & Ciência \& Saúde Coletiva \\
\hline 18 & $\begin{array}{l}\text { O SUS e o setor privado assistencial: } \\
\text { interpretações e fatos }\end{array}$ & $\begin{array}{l}\text { Bahia e Scheffer } \\
(2018)\end{array}$ & Saúde em Debate \\
\hline 19 & $\begin{array}{l}\text { Organizações Sociais da Saúde: uma expressão } \\
\text { fenomênica da privatização da saúde no Brasil }\end{array}$ & Moraes et al. (2018) & Cadernos de Saúde Pública \\
\hline 20 & $\begin{array}{l}30 \text { anos de SUS: relação público-privada e os } \\
\text { impasses para o direito universal à saúde }\end{array}$ & Celuppi et al. (2019) & Saúde em Debate \\
\hline 21 & $\begin{array}{l}\text { Reflexões de médicos sobre o trabalho na } \\
\text { Estratégia de Saúde da Família sob a gestão } \\
\text { das Organizações Sociais }\end{array}$ & Ballarotti et al. (2019) & $\begin{array}{l}\text { Interface - Comunicação, } \\
\text { Saúde, Educação }\end{array}$ \\
\hline 22 & $\begin{array}{l}\text { A judicialização da saúde no Brasil e na } \\
\text { Colômbia: uma discussão à luz do novo } \\
\text { constitucionalismo latino-americano }\end{array}$ & $\begin{array}{l}\text { d'Ávila, Andrade e } \\
\text { Aith (2020) }\end{array}$ & Saúde e Sociedade \\
\hline 23 & \begin{tabular}{|l|} 
Nenhum Serviço de Saúde a Menos: \\
movimentos sociais, novos sujeitos políticos e \\
direito à saúde em tempos de crise no Rio de \\
Janeiro, Brasil
\end{tabular} & $\begin{array}{l}\text { Saffer, Mattos e Rego } \\
(2020)\end{array}$ & Saúde em Debate \\
\hline 24 & $\begin{array}{l}\text { Neoliberalism in Latin America: effects on } \\
\text { health system reforms }\end{array}$ & $\begin{array}{l}\text { Gottems e Mollo } \\
(2020)\end{array}$ & Revista de Saúde Pública \\
\hline 25 & $\begin{array}{l}\text { Previne Brasil, Agência de Desenvolvimento } \\
\text { da Atenção Primária e Carteira de Serviços: } \\
\text { radicalização da política de privatização da } \\
\text { atenção básica? }\end{array}$ & $\begin{array}{l}\text { Morosini, Fonseca e } \\
\text { Baptista (2020) }\end{array}$ & Cadernos de Saúde Pública \\
\hline 26 & $\begin{array}{l}\text { Quando os sistemas públicos de saúde são } \\
\text { notícia: uma análise comparativa da cobertura } \\
\text { jornalista no Brasil e na Espanha }\end{array}$ & $\begin{array}{l}\text { Langbecker, } \\
\text { Castellanos e } \\
\text { Matamoros (2020) } \\
\end{array}$ & Ciência \& Saúde Coletiva \\
\hline 27 & $\begin{array}{l}\text { Trajetória da privatização do sistema de saúde } \\
\text { chileno (1924-2005) }\end{array}$ & $\begin{array}{l}\text { Cortés, Coelho e } \\
\text { Sanabria (2020) }\end{array}$ & Saúde em Debate \\
\hline
\end{tabular}

Fonte: Autores a partir da base de dados da Scielo. 
Para complementar o estudo bibliométrico, tem sido recorrente a utilização das ferramentas associadas à Análise de Redes Sociais (ARS), devido a possibilidade de se apresentar o conhecimento de forma visual, bem como identificar elementos críticos para a conformação de determinado conhecimento científico sobre o objeto de estudo (Van Eck, 2014).

Desta forma, o presente artigo utiliza a ARS para: i) construção da rede de interação entre os artigos da amostra e os grupos de análise dos elementos privatizantes do SUS; e ii) construção da rede de palavras-chave dos referidos artigos científicos. O software utilizado para visualização das redes citadas é o gephi (https://gephi.org/), em sua última versão disponível, utilizando a métrica de centralidade de autovetor ${ }^{1}$ para identificar atores relevantes das redes (Bastian, Heymann \& Jacomy, 2009).

Ademais, realizou-se uma análise qualitativa dos artigos constantes da amostra aglutinando por grupos de fatores relacionados com o estudo da privatização do SUS, identificando elementos mais abordados e fatores privatizantes do SUS que podem ser elementos de estudos futuros.

\section{Resultados e Discussão}

\subsection{Resultados de Métricas Bibliométricas}

$\mathrm{Na}$ perspectiva temporal da amostra dos artigos que tratam sobre os elementos relacionados com a privatização da saúde pública no Brasil, o primeiro artigo foi publicado em 1999, sendo a maior parte das publicações concentradas no período de 2011 a 2020, onde 23 dos 27 artigos da amostra foram publicados nessa última década.

Esta concentração de publicações nesta última década revela a intensificação da discussão e das reflexões sobre o tema. Além disso, boa parte dos artigos do período 2011-2020 estão concentrados em períodos recentes, a partir de 2016. Abaixo, segue o Gráfico 1, que apresenta a evolução temporal dos artigos da amostra.

Gráfico 1: Evolução de artigos publicados sobre a privatização do SUS, nos períodos de 1991-2000, 2001-2010 e 2011-2020.

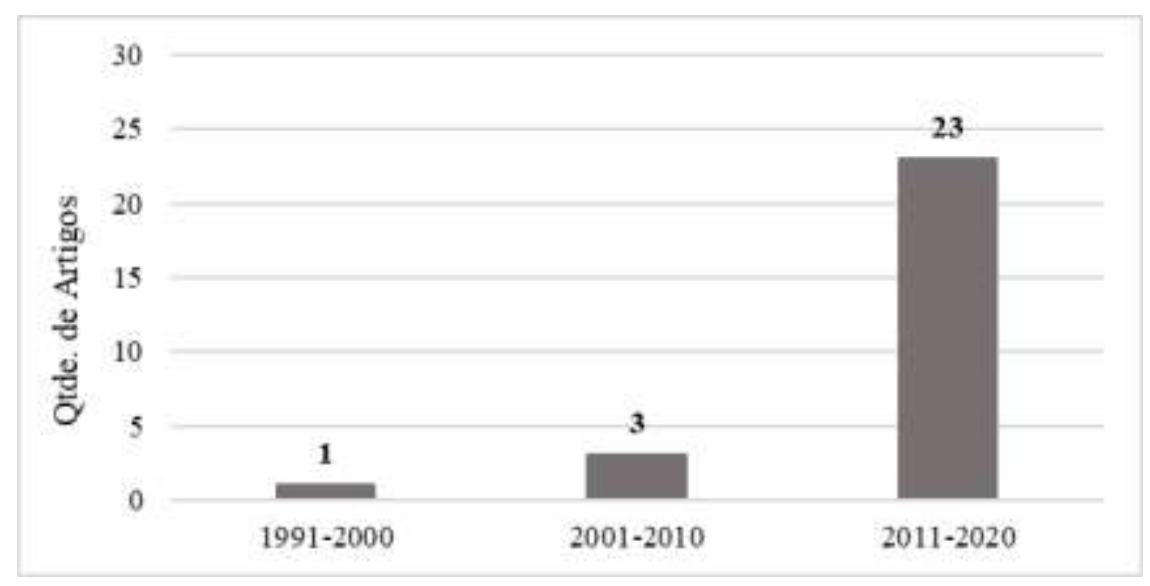

Fonte: Autores a partir da base de dados da Scielo.

A partir da análise dos artigos da amostra, foram identificados uma quantidade de 62 autores para o conjunto dos 27 artigos, representando uma média para amostra de 2,30 autores por artigo. Sobre as instituições associadas nos referidos artigos, a Fundação Oswaldo Cruz (FIOCRUZ) é a principal instituição associada aos autores da referida amostra, no qual $17,74 \%$ dos autores estão associados à referida instituição.

Além disso, ao observar as sete principais instituições que estão associadas aos autores que publicaram artigos relacionados com os elementos privatizantes do SUS - além da FIOCRUZ, Universidade de São Paulo (USP), Universidade

\footnotetext{
${ }^{1}$ Segundo Borgatti (2005), a centralidade de autovetor indica a intensidade de conexão de um agente com os agentes mais relevantes da rede.
} 
Federal do Pernambuco (UFPE), Universidade Federal da Fronteira Sul (UFFS), Pontifícia Universidade Católica de São Paulo (PUC-SP), Universidade Estadual de Feira de Santana (UEFS) e Universidade Estadual do Oeste do Paraná (UNIOESTE)estão associados em conjunto com $56,45 \%$ dos autores da referida amostra. As informações estão apresentadas na Tabela 1 abaixo.

Tabela 1: Instituições associadas aos autores dos artigos relacionados com os elementos privatizantes do SUS.

\begin{tabular}{|c|c|c|}
\hline Instituição & Qtde. Autores & \%. Part. \\
\hline FIOCRUZ & 11 & $17,74 \%$ \\
\hline USP & 6 & $9,68 \%$ \\
\hline UFPE & 5 & $8,06 \%$ \\
\hline UFFS & 4 & $6,45 \%$ \\
\hline PUC-SP & 3 & $4,84 \%$ \\
\hline UEFS & 3 & $4,84 \%$ \\
\hline UNIOESTE & 3 & $4,84 \%$ \\
\hline Top 7 & 35 & $56,45 \%$ \\
\hline Outras Instituições & 27 & $43,55 \%$ \\
\hline Total Geral & 62 & $100,00 \%$ \\
\hline
\end{tabular}

Fonte: Autores, a partir da base de dados da Scielo.

Quanto as revistas científicas associadas aos artigos da amostra, verifica-se que as quatro principais revistas em termos de artigos publicados que se relacionam com a discussão da privatização do SUS, são Cadernos de Saúde Pública, Ciência \& Saúde Coletiva, Saúde em Debate e Saúde e Sociedade, que em conjunto, concentram 59,25\% dos artigos da amostra. Destas revistas científicas, a que possui maior quantidade de publicações é a Cadernos de Saúde Pública, com 5 artigos da amostra, representando 18,52\% dos artigos relacionados com os estudos sobre elementos privatizantes do SUS.

Quanto ao indicador de fator de impacto da revista, o presente estudo utiliza como proxy o h5 index do Google Scholar Metrics. Está métrica busca medir o impacto dos artigos publicados no Google Scholar, sendo uma alternativa interessante à utilização dos indicadores do Fator de Impacto (FI) do Journal of Citation Reports (JCR) e do CiteScore do Scimago Journal \& Country Rank (SJR) para as revistas científicas nacionais (Zhang \& Lun, 2019; Costa, Canto \& Pinto, 2020). O h5 index "se refere ao número h de um periódico, em que h artigos publicados nos últimos cinco anos tenham sido citados no mínimo h vezes cada" (Costa, Canto \& Pinto, 2020, p. 7).

Neste sentido, os periódicos que possuem os maiores h5 index da amostra são Ciência \& Saúde Coletiva, Revista de Saúde Pública e Cadernos de Saúde Pública, com h5 index para o ano de 2020 de 49, 41 e 40, respectivamente. Da referida amostra, as revistas Psicologia \& Sociedade (19), História, Ciências, Saúde-Manguinhos (16) e Revista Katálysis (10) possuem os menores h5 index. Ainda sobre o referido indicador de impacto dos periódicos da amostra, o h5 index médio é de 26,54, no qual 7 das 13 revistas listadas na Tabela 2 possuem um h5 inferior à média. 
Tabela 2: Lista das revistas científicas dos artigos da amostra.

\begin{tabular}{|c|c|c|c|c|}
\hline Revista Científica & Artigos & $\%$ part. & ISSN & h5 index (2020) \\
\hline Cadernos de Saúde Pública & 5 & $18,52 \%$ & $0102-311 X$ & 40 \\
\hline Ciência \& Saúde Coletiva & 4 & $14,81 \%$ & $1413-8123$ & 49 \\
\hline Saúde em Debate & 4 & $14,81 \%$ & 0103-1104 & 28 \\
\hline Saúde e Sociedade & 3 & $11,11 \%$ & 0104-1290 & 27 \\
\hline Revista Katálysis & 2 & $7,41 \%$ & $1414-4980$ & 10 \\
\hline Trabalho, Educação e Saúde & 2 & $7,41 \%$ & $1981-7746$ & 20 \\
\hline Brazilian Journal of Political Economy & 1 & $3,70 \%$ & $0101-3157$ & 22 \\
\hline Physis: Revista de Saúde Coletiva & 1 & $3,70 \%$ & $0103-7331$ & 22 \\
\hline EURE (Santiago) & 1 & $3,70 \%$ & $0250-7161$ & 21 \\
\hline História, Ciências, Saúde-Manguinhos & 1 & $3,70 \%$ & 0104-5970 & 16 \\
\hline Interface - Comunicação, Saúde, Educação & 1 & $3,70 \%$ & $1414-3283$ & 30 \\
\hline Psicologia \& Sociedade & 1 & $3,70 \%$ & $1807-0310$ & 19 \\
\hline Revista de Saúde Pública & 1 & $3,70 \%$ & $1518-8787$ & 41 \\
\hline Total & 27 & & Média h5 Index & 26,54 \\
\hline
\end{tabular}

Fonte: Fonte: Autores, a partir da base de dados da Scielo.

Ainda sobre os periódicos associados aos artigos da amostra, ao observar as áreas de concentração das referidas revistas científicas, a área da saúde coletiva é a mais representativa para os estudos relacionados com os elementos que incentivam a privatização de um sistema de saúde concebido de forma pública e universal, sendo direcionado cada vez mais para um controle privado e mercantilista. Outras áreas como a economia, serviço social e a psicologia se revelam, de forma minoritária, partes interessadas nos estudos das causas e consequências dos fatores que incentivam a privatização do SUS sobre a sociedade.

\subsection{Análise de Redes Sociais: Rede entre artigos científicos da amostra e os fatores relacionados com a privatização do} SUS e Rede de palavras-chave

Para a análise dos artigos da amostra apresentados no Quadro 2 na seção metodológica, com o intuito de sistematizar a exposição dos resultados, este trabalho propõe a segregação dos fatores que evidenciam o processo de privatização do SUS em 6 (seis) grupos de análise.

A organização dos artigos científicos da amostra em grupos de análise consiste na identificação dos principais pontos discutidos até o presente momento quanto ao movimento ou a existência de ações que direcionam na alteração da estrutura da política de saúde no país em divergência às bases constituídas na reforma sanitária e na Constituição Federal de 1988. Assim, será possível apontar os pontos mais e menos discutidos, de forma a subsidiar o desenvolvimento de estudos futuros.

Desta forma, segue abaixo os seis grupos de análise relacionados com os elementos que evidenciam os movimentos em direção à privatização do SUS:

i. Marco Político-Institucional: associado ao regime de incentivos e políticas do Estado, além da redefinição do ambiente institucional, criando elementos que favoreçam a concepção de saúde numa perspectiva individual ou privada;

ii. $\quad$ Financiamento: ações e políticas que alterem a destinação dos recursos para o setor saúde, bem como 
mecanismos de restrição orçamentária;

iii. Gestão: relacionado à execução das diretrizes estabelecidas na política de saúde e associado à capacidade de administrar e gerenciar recursos, organizações e instituições;

iv. Participação Social: relacionado com as formas de participação da sociedade civil e do canal de comunicação entre a sociedade e os agentes representativos do Estado, de forma a subsidiar no desenvolvimento de políticas públicas de saúde mais aderentes à realidade local;

v. Análise Comparada: realização de comparação entre políticas de saúde entre países, de forma a subsidiar interpretações sobre a realidade nacional; $\mathrm{e}$

vi. Formação Profissional e Relações de Trabalho: relacionado com a formação profissional das atividades relacionadas à saúde, bem como as relações de trabalho nas instituições integrantes na prestação de serviço de saúde.

Em uma concepção neoliberal, a necessidade de reorganização do Estado para uma nova forma de atuação para provisão de bens e serviços à sociedade se faz necessária. É neste sentido que as análises dos elementos privatizantes do SUS estão associados ao Marco Político-Institucional, tendo em vista que para este novo enquadramento na atuação estatal, são exigidas inovações organizacionais e institucionais para sua promoção.

Ademais, a lógica neoliberal para as políticas de saúde está relacionada com o predomínio das regras da concorrência e da redução dos custos econômicos, diametralmente oposto com a organização e concepção dos sistemas universais de saúde (Gottems \& Mollo, 2020). Em contextualização, Campos, Viana e Soares (2015) apresentam as formas de reorganização das políticas estatais no Brasil na década de 1990, sendo direcionadas para uma

“(...)"reestruturação produtiva”, com a corrosão dos direitos trabalhistas e outras ações (incluindo a criação dos "contratos temporários", o aumento do tempo para aposentadoria etc.), cortes nos gastos estatais, privatização, sujeição do público ao privado (tanto no nível da prioridade da ação estatal quanto em propostas de "parceria", que apontam para maior privatização e mercantilização do estatal), políticas financeiras de ajuste, políticas de assistência social paliativas em substituição às políticas estruturais etc” (Campos, Viana \& Soares, 2015, p. 86).

Neste sentido, Costa (2008) evidencia a importância da criação da Agência Nacional de Saúde Suplementar (ANS) para a organização de um mercado privado de saúde no Brasil, relacionado aos planos de saúde. Tal fato está associado à concepção da reforma do Estado e a mudança na sua atuação, direcionando a ação do Estado para regulação ao invés de produção de bens e serviços para a sociedade.

Para Souza (2014), ao longo da década de 1990, ainda associado com a redefinição do papel do Estado, verificou-se que ao longo da vida do SUS foram implementadas diversas ações que divergem da concepção universalista, com a adoção de políticas públicas focalizadas, redirecionando as ações no SUS, com uma orientação do Estado para a política social residual, favorecendo a elevação da participação do setor privado na prestação de serviço de saúde à população (Cêa, Reis \& Conterno, 2007; Campos, Viana \& Soares, 2015; Marques, Ximenes \& Ugino, 2018), criando um ambiente propício para a negação de direitos estabelecidos na carta magna (Costa, 2014; d'Ávila, Andrade \& Aith, 2020).

Para Bahia \& Scheffer (2018), a participação de empresários de saúde e de seus representantes em partidos políticos, bem como a utilização por parte das lideranças políticas das mais diversas matizes ideológicos de atendimentos em hospitais particulares podem evidenciar a compreensão da classe política do "senso comum sobre a capacidade de estratégias individualizadas responderem a problemas de saúde que são coletivos” (Bahia \& Scheffer, 2018, p. 169).

Assim, a construção política também será direcionada na compreensão da superioridade de eficiência do setor privado 
em relação ao setor público, indicando que sistemas universais de saúde seriam pouco relevantes para mitigação de desigualdades (Bahia \& Scheffer, 2018). Neste sentido, tais elementos fazem com que a política de saúde praticada no Brasil atualmente seja caracterizada pela convivência de elementos dicotômicos, possuindo características universalistas e focalistas ao mesmo tempo (Marques, Ximenes \& Ugino, 2018), além de estar relacionado com a disputa de poderes nas esferas política, social e econômica na relação público-privada no sistema de saúde (Celuppi et al., 2019).

Quanto ao aspecto do financiamento do SUS, as evidências listadas estão relacionadas ao incentivo por parte do Estado em relação ao consumo privado de saúde, como o abatimento desses gastos relacionas aos planos de saúde no imposto de renda para pessoa física, seja o trabalhador da iniciativa privada ou do setor público, gerando a renúncia de receita e aprofundando o seu subfinanciamento (Contreiras \& Matta, 2015; Bahia \& Scheffer, 2018; Marques, Ximenes \& Ugino, 2018; Sposati, 2018; Gottems \& Mollo, 2020).

O subfinanciamento da saúde pública do país acaba por ser potencializada em um ambiente em que a União e os entes federativos são submetidos às políticas de austeridade fiscal que impedem a execução da prestação de serviço justo e de qualidade (Valla, 1998; Campos, Viana \& Soares, 2015; Celuppi et al., 2019). Em um país continental com desigualdades regionais importantes como o Brasil, tais políticas restritivas que reduzem o acesso ao sistema de saúde de forma universal e gratuita acabam por aprofundar tais desigualdades regionais (Pimenta \& Pimenta, 2011; Morosini, Fonseca \& Baptista, 2020).

Ademais, cabe salientar o advento da lei de responsabilidade fiscal, limitando os gastos em pessoal pelos entes federativos, representando o engessamento para a área da saúde quanto ao direcionamento de recursos em um setor intensivo em capital humano (Noronha et al., 2018; Celuppi et al., 2019). A desvinculação das receitas da União direcionados para a área da saúde, com o direcionamento orçamentário para outras finalidades dificultam a execução dos serviços essenciais (Noronha et al., 2018).

Mais recentemente, o aprofundamento do subfinanciamento do SUS está relacionado com a Emenda Constitucional no 95/2016, que congela os gastos primários pelo período de 20 anos. Segundo Celuppi et al. (2019), esta emenda consiste na “(...) retração do financiamento do sistema de saúde brasileiro, que se torna um agravante ainda maior para a sustentabilidade e manutenção do SUS. A perda significativa de recursos proposta por essa medida obriga os gestores e secretários a reduzirem as ações e serviços disponíveis aos usuários, sob o preceito de diminuir as despesas dos estados e municípios" (Celuppi et al., 2019, p. 308).

Quanto às evidências de elementos privatizantes do SUS relacionadas à gestão, a concepção da criação de um setor público não-estatal está em consonância com a reforma administrativa do Estado, sendo influenciado diretamente pela concepção neoliberal, no qual a constituição de um setor privado "por dentro" do setor público estaria associado ao movimento de modernização e eficiência gerencial (Cêa, Reis \& Conterno, 2007; Lara, Guareschi \& Bernardes, 2016).

Os artigos científicos relacionados com os aspectos de gestão colocam a institucionalização e pulverização das Organizações Sociais (OSs), Organizações da Sociedade Civil de Interesse Público (OSCIPs) e Parcerias Público-Privadas na gestão de unidades de saúde como um elemento importante para a redefinição do setor público na prestação de serviços de saúde (Sousa, 2014; Campos, Viana \& Soares, 2015; Contreiras \& Matta, 2015; Druck, 2016 Morais et al., 2018; Ballatori et al., 2019; Morosini, Fonseca \& Baptista, 2020).

Ainda sobre os aspectos relacionados à Gestão, a possibilidade de se criar Fundações Estatais de Direito Privado (FEDP) é apresentada como uma alternativa para a flexibilização e agilidade na gestão dos serviços de saúde. A criação da Empresa Brasileira de Serviços Hospitalares (EBSERVH), além da existência de OSs e OSCIPs, eleva o conjunto de possibilidades das unidades de saúde para estabelecer a gestão dos serviços de saúde pública de forma não-estatal (Druck, 2016). Além disso, o atendimento de pacientes com planos de saúde em um estabelecimento público- ocorrendo a "dupla entrada" no atendimento- insere uma lógica de acumulação de capital e do lucro, em um sistema constituído sobre bases 
universais, impactando também no ensino médico, ao se tratar de hospitais universitários (Silva \& Caxias, 2016).

Outro ponto a destacar é a aprovação da Lei $n^{\circ} 13.097 / 2015$, que permite a participação de capital estrangeiro nos segmentos relacionados à assistência à saúde. Segundo Marques, Ximenes e Ugino (2018) e Celuppi et al. (2019), esta lei abre espaço para a criação de um setor privado de saúde ainda mais concentrado, favorecendo o fortalecimento de mecanismos de influência e de relações de poder sobre o setor público e seus usuários por parte dos grandes conglomerados internacionais, sendo este mais um mecanismo que restringe a consolidação e a afirmação do SUS sob a perspectiva universal.

No que diz respeito aos pontos relacionados com a participação social, Valla (1998) apresenta uma questão importante sobre a relação entre os mediadores e as classes populares, quanto a capacidade destes mediadores de interpretar as reais necessidades e reivindicações apresentadas por manifestações populares. Cabe ressaltar que um canal importante de participação social e mediação de interesses na Saúde são os Conselhos Municipais de Saúde.

Neste sentido, tendo em vista a pouca confiabilidade da população sobre a atividade política, existe pouca relevância para as classes populares em mudanças associadas a atividade parlamentar e negociações e reivindicações junto às autoridades estabelecidas (Valla, 1998). Assim, são apresentadas as tendências de participação popular proeminentes na realidade brasileira, sendo uma delas apoiada sob a perspectiva de solidariedade, apoiadas por ONGs, Igrejas e Associação de Moradores, e outra apoiada na prática de ocupação ou acontecimentos consumados como forma de estabelecimento de canal de negociação, tendo como o exemplo o Movimento dos Trabalhadores Rurais Sem Terra (MST) (Valla, 1998).

Santos (2013) expõem que há uma restrição na comunicação e participação entre a sociedade civil e o Estado, sendo limitado ainda aos trabalhadores da saúde, muito embora a gestão participativa seja uma das bases que estrutura o SUS fundamentada na relação entre o estado, os trabalhadores da saúde e a sociedade civil. Esta restrição e fragilização dos espaços de participação democrática também estão relacionados com a afirmação da lógica neoliberal sobre as políticas sociais.

Um dos elementos privatizantes do SUS se deve ao baixo engajamento dos sindicatos no que diz respeito às bases da reforma sanitária e na constituição de um sistema público genuinamente universal, "mergulhado nas contradições entre a postura ideológica igualitária e a defesa de interesses corporativos" (Costa, 2014, p. 822). Relacionado a isto, o que se verifica de forma concomitante ao estabelecimento do SUS é o elevado interesse por parte dos sindicatos no incentivo ao mercado privado de saúde ao colocar em pauta nos acordos coletivos planos de saúde para os trabalhadores (Costa, 2014; Bahia \& Scheffer, 2018).

Além disso, o argumento utilizado de forma corrente pela base sindical reside em que, na medida que determinado grupo de trabalhadores e seus respectivos dependentes tenham acesso a planos de saúde corporativos, estes estariam “desafogando" o sistema público de saúde para atender prioritariamente os mais pobres (Bahia \& Scheffer, 2018). Este tipo de situação gera uma adesão e concordância indireta por parte de sindicatos quanto à políticas focalistas, políticas estas que caminham em conjunto com ações que visam restringir e retirar direitos dos trabalhadores.

Além disso, Saffer, Mattos e Rego (2015) ressaltam que a adoção de modalidades de gestão como OS possui pouca aderência no que diz respeito à questão da participação e mecanismos de controle social. Assim, o baixo engajamento da sociedade na defesa de um sistema genuinamente público e universal, associado com mecanismos de gestão privada, ocasiona o aprofundamento do déficit de controle e participação social quanto à política de saúde.

Quanto à questão da análise comparada, o artigo de Garrido-Pinzón e Bernardo (2017) estuda as vivências de trabalhadores da atenção básica dos sistemas públicos de saúde da Colômbia e do Brasil, sob a ótica neoliberal. Langbecker, Castellanos e Matamorros, estudam a cobertura jornalística sobre o SUS, na "Folha de São Paulo", e sobre o Sistema Nacional de Salud (SNS - Espanha), no "El País".

A análise da judicialização da saúde no Brasil e na Colômbia à luz do novo constitucionalismo latino-americano é objeto de estudo comparado de d'Ávila, Andrade e Aith (2020). Cortéz, Coelho e Sanabria (2020) estudam a trajetória da 
privatização do sistema de saúde do Chile para auxiliar na compreensão dos elementos privatizantes do SUS. Gottems \& Mollo estudam os efeitos das políticas neoliberais nas reformas dos sistemas de saúde da Argentina, Brasil, Chile, Colômbia, México e Peru.

Em relação a categoria de análise relacionado com a formação profissional e relações de trabalho, Cêa, Reis e Conterno (2007) evidenciam o Projeto de Profissionalização dos Trabalhadores da Área de Enfermagem (Profae) estabelecido pelo ministério da saúde, como um programa constituído sob influência dos mecanismos de reforma do estado em um ambiente de adoção de políticas neoliberais. Bispo Júnior (2009) estuda as condições de expansão do ensino e formação em fisioterapia no Brasil, apresentando a necessidade do ensino e formação em fisioterapia de se distanciar do modelo de formação curativo-reabilitador privatista, com a sua aproximação com os preceitos e diretrizes do SUS e adequar a formação dos profissionais ao perfil epidemiológico da população (Bispo Júnior, 2009).

Drucker (2016) verifica que a introdução destas organizações na prestação de serviços públicos de saúde aprofunda o processo de terceirização e da precarização dos postos de trabalho na área da saúde, sendo verificadas remunerações inferiores às praticadas pelo servidor público, além do estabelecimento de relações frágeis de trabalho em um setor de caráter continuado (Bahia \& Scheffer, 2018; Garrido-Pinzón \& Bernardo, 2017; Ballarotti et al., 2019).

Após toda a descrição analítica, a partir dos artigos científicos da amostra e dos grupos de análise relacionados com os elementos privatizantes do SUS, foi possível construir uma rede interativa entre os artigos científicos e os grupos de análise sobre a privatização do SUS, de forma a identificar os grupos de análise mais e menos representativos entre os artigos científicos e os artigos científicos com maior diversidade de análise dos elementos privatizantes do SUS. Na perspectiva dos artigos científicos da amostra, os artigos de Sousa (2014), Campos, Viana e Soares (2015) e Marques, Ximenes e Ugino (2018) apresentam os maiores scores de autovetor dentre as publicações constantes na rede.

Quanto aos grupos de análise, considerando o score de centralidade de autovetor extraído da rede entre os artigos científicos e os grupos de análise sobre a privatização do SUS, conforme apresentado na Figura 2, os principais grupos de análise tratados em boa parte dos artigos científicos são fatores ligados à gestão, financiamento e marco político-institucional, com scores de autovetor no valor de 1, 0,98 e 0,82, respectivamente. Tais pontos representam os elementos mais estudados pelos artigos científicos quanto ao processo de privatização do SUS. 
Figura 2: Rede de interação entre os artigos científicos da amostra e os fatores relacionados com a privatização do SUS (*).

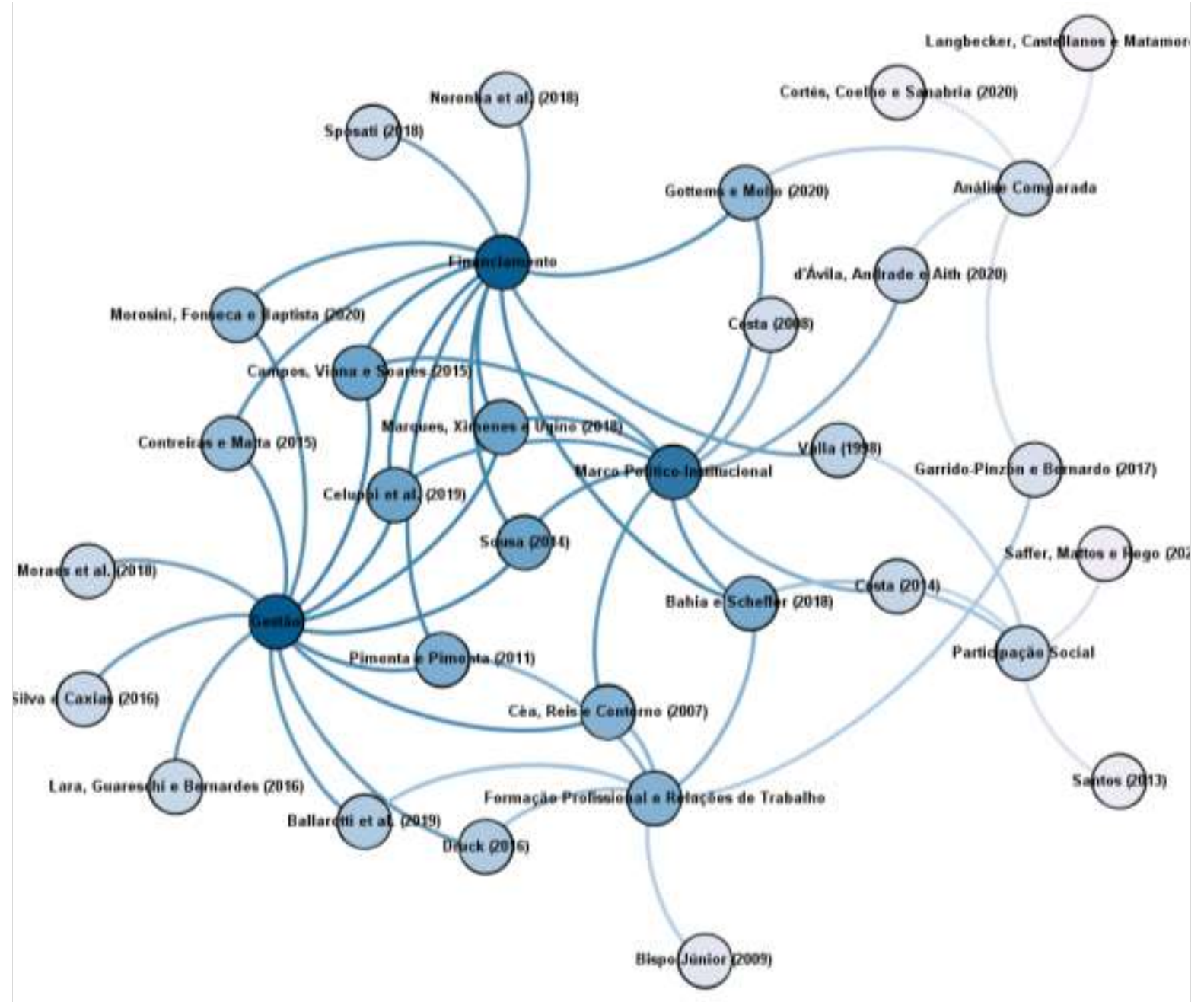

Nota (*): quanto maior a intensidade das cores, maior é o score de centralidade de autovetor. Fonte: Elaboração dos autores.

Já os grupos relacionados com a formação profissional e relações de trabalho, participação social e análise comparada possuem os menores scores de autovetor dentre os grupos de análise, no valor de 0,45, 0,22 e 0,18, respectivamente, indicando que estes temas são relativamente pouco abordados.

Neste sentido, tais temas podem representar um caminho interessante para o desenvolvimento de novos estudos relacionados com os elementos privatizantes do SUS e preenchimento de lacunas possivelmente existentes, tendo em vista que, por exemplo, a utilização da análise comparada para entender os elementos de privatização da saúde no Brasil a partir de experiências comparadas é verificada em estudos recentes, a partir do ano de 2017.

Quanto as principais palavras-chave dos artigos que discutem de forma direta e indiretamente as questões relacionadas com a privatização da saúde no Brasil, os principais termos utilizados e seus respectivos scores de autovetor são SUS (1,00), privatização $(0,76)$, política de saúde $(0,72)$, reforma dos serviços de saúde $(0,57)$, neoliberalismo $(0,54)$, Brasil $(0,52)$, sistemas de saúde $(0,48)$, gestão em saúde $(0,48)$, direito à saúde $(0,44)$ e atenção primária em saúde $(0,41)$. A Rede de palavras-chave está apresentada na Figura 3. 
Figura 3: Rede de palavras-chave dos artigos científicos da amostra (*).

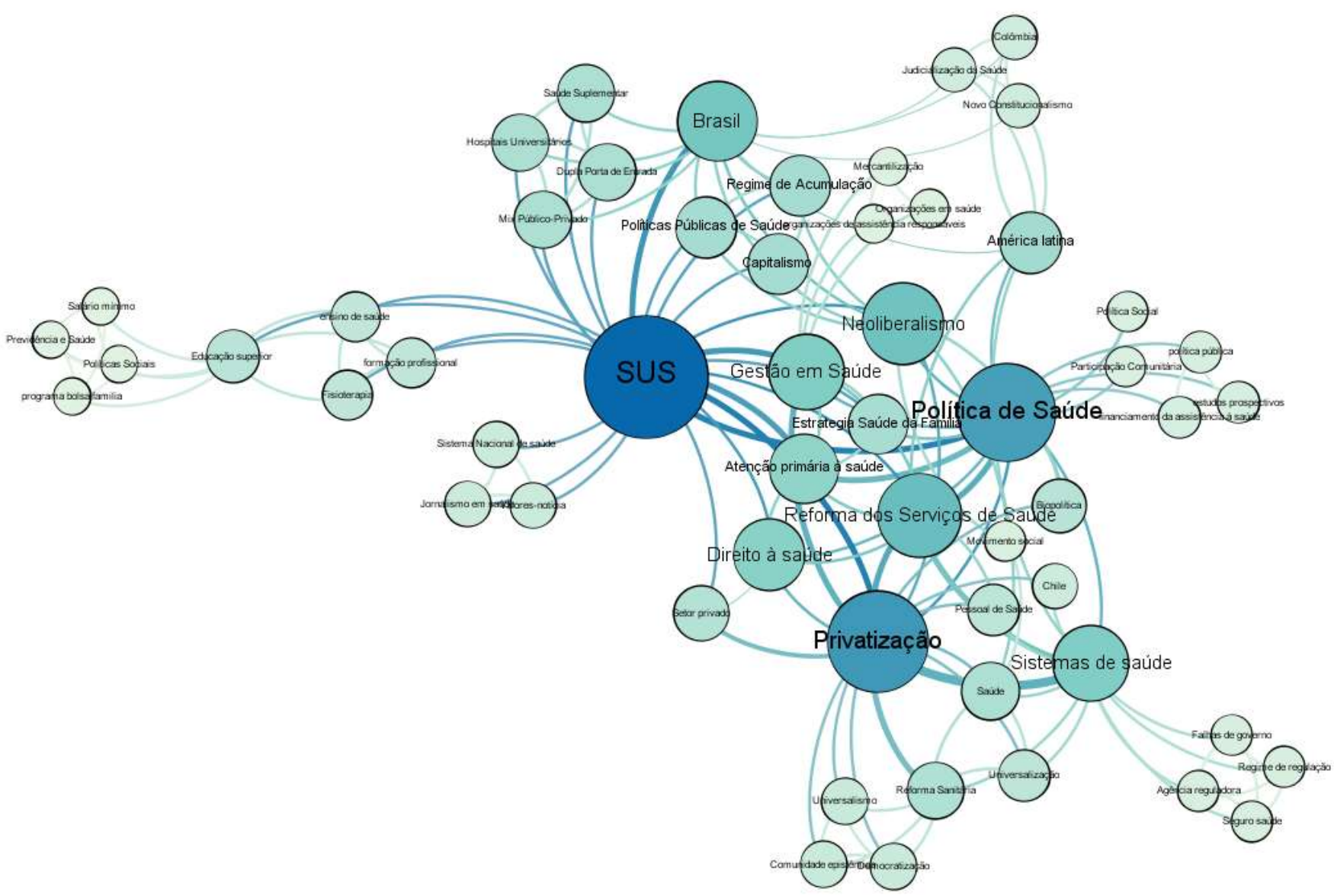

Nota (*): quanto maior a intensidade das cores e o tamanho do nó, maior é o score de centralidade de autovetor Fonte: Autores.

Tais palavras-chave encontradas estão, em boa medida, relacionadas com o tema em discussão. Além dos termos utilizados para os protocolos de busca dos artigos constante no Quadro 1, que em sua maioria estão contempladas entre as principais palavras-chave, outras palavras utilizadas marcadas com elevada frequência entre os artigos da amostra são importantes para indicar o desenvolvimento teórico.

As palavras-chave "política de saúde" e "reforma dos serviços de saúde" indicam a influência das políticas de saúde no Brasil e na américa latina sob a perspectiva neoliberal, sobretudo em relação à dicotomia entre o público e o privado no atendimento das demandas relacionadas à saúde. A palavra "sistemas de saúde" reforça a questão da análise comparada entre experiências de sistemas de saúde para análise dos elementos privatizantes da saúde pública. No caso das palavras "gestão em saúde" e "atenção primária em saúde", reforçam os aspectos relacionados com a gestão como um dos elementos relevantes para o estudo da privatização do SUS. Em um ambiente de políticas sociais focalizadas e com restrições orçamentárias relevantes, a palavra "direito à saúde" pode indicar a necessidade de afirmação da saúde pública na perspectiva universal, sendo o ambiente atual propício para o estabelecimento da situação de negação de direito à saúde.

\section{Considerações Finais}

O objetivo do artigo foi a realização da revisão bibliográfica sobre os estudos relacionados com a privatização do Sistema Único de Saúde (SUS) no Brasil, organizando o conhecimento científico estabelecido sobre o referido tema e indicar possíveis lacunas e caminhos existentes para o desenvolvimento científico sobre um assunto relevante para a sociedade, 
sobretudo no contexto atual, uma vez que no momento em que este artigo é confeccionado, os países enfrentam um desafio imenso relacionado à Pandemia do SARS-CoV-2 (COVID-19).

Para a revisão bibliográfica sobre os elementos privatizantes do SUS, foi utilizada a base de dados da Scielo como referência de coleta e busca dos artigos científicos relacionados ao tema. Utilizou-se também algumas métricas bibliométricas para o presente estudo, como a evolução temporal dos artigos científicos, identificação das principais: i) instituições associados aos autores dos artigos; e ii) principais revistas dos artigos da amostra. Houve também a utilização da análise de redes sociais para a construção da análise de rede de palavras chave e a análise de rede entre os artigos da amostra e os grupos de análise dos fatores que constituem em elementos que direcionam para a privatização do SUS.

Assim, identificou-se que o primeiro artigo publicado relacionado direta ou indiretamente com a privatização da saúde no Brasil foi em 1998, a maior parte dos artigos publicados se concentram no período de 2011 a 2020 e que os artigos da amostra possuem em média, 2,30 autores por artigo. A Fundação Oswaldo Cruz (FIOCRUZ) é a principal instituição associada aos autores da referida amostra, no qual 17,74\% dos autores dos artigos em questão. Além disso, as sete principais instituições relacionadas aos autores dos artigos da amostra concentram $56,45 \%$ dos autores.

As principais revistas científicas identificadas em termos de quantidade de artigos publicados e em relação ao h5 index são Cadernos de Saúde Pública e Ciência \& Saúde Coletiva, respectivamente. Observa-se entre as revistas científicas a relevância da área de saúde coletiva para os estudos sobre a privatização do SUS. De forma minoritária, observa-se contribuições da economia, serviço social e da psicologia para o desenvolvimento científico sobre o tema.

Sobre a rede de palavras-chave, identificou-se os termos SUS, privatização, política de saúde, reforma dos serviços de saúde, neoliberalismo, Brasil, sistemas de saúde, gestão em saúde, direito à saúde e atenção primária em saúde, como as palavras-chave de com as maiores ocorrências dentre os artigos da amostra.

Entre a rede de entre os artigos e os grupos de análise dos elementos privatizantes do SUS, identificou-se que os grupos de análise gestão, financiamento e marco político-institucional são os mais estudados pelos artigos científicos quanto ao processo de privatização do SUS. Por outro lado, os grupos relacionados com a formação profissional e relações de trabalho, participação social e análise comparada são pouco abordados pelos artigos da amostra.

Neste sentido, um caminho interessante para o desenvolvimento de estudos futuros relacionados com os elementos privatizantes do SUS é o aprofundamento científico nas questões relacionadas com a formação profissional e relações de trabalho, participação social e análise comparada. Tais estudos são relevantes para a melhor caracterização dos processos estabelecidos com vistas a subsidiar políticas públicas que reforcem a concepção da saúde pública no país como um direito e de acesso universal.

\section{Referências}

Bahia, L., \& Scheffer, M. (2018). O SUS e o setor privado assistencial: interpretações e fatos. Saúde em Debate, 42, $158-171$.

Ballarotti, B., Corneau, F. G., Junqueira, V., \& Mendes, Á. (2019). Reflexões de médicos sobre o trabalho na Estratégia de Saúde da Família sob a gestão das Organizações Sociais. Interface-Comunicação, Saúde, Educação, 23, e180082.

Bastian M., Heymann S., \& Jacomy M. (2009). Gephi: an open source software for exploring and manipulating networks. International AAAI Conference on Weblogs and Social Media

Bispo Júnior, J. P. (2009). Formação em fisioterapia no Brasil: reflexões sobre a expansão do ensino e os modelos de formação. História, Ciências, SaúdeManguinhos, 16(3), 655-668.

Borgatti, S. P. (2005). Centrality and network flow. Social networks, 27(1), 55-71.

Campos, C. M. S., Viana, N., \& Soares, C. B. (2015). Mudanças no capitalismo contemporâneo e seu impacto sobre as políticas estatais: o SUS em debate. Saúde e Sociedade, 24, 82-91.

Cêa, G. S. D. S., Reis, L. F., \& Conterno, S. (2007). Profae e lógica neoliberal: estreitas relações. Trabalho, Educação e Saúde, 5(1), 139-160. 
Celuppi, I. C., Geremia, D. S., Ferreira, J., Pereira, A. M. M., \& Souza, J. B. D. (2019). 30 anos de SUS: relação público-privada e os impasses para o direito universal à saúde. Saúde em Debate, 43, 302-313.

Contreiras, H., \& Matta, G. C. (2015). Privatização da gestão do sistema municipal de saúde por meio de Organizações Sociais na cidade de São Paulo, Brasil: caracterização e análise da regulação. Cadernos de Saúde Pública, 31, 285-297.

Cortés, F. A. M., Coelho, T. C. B., \& Sanabria, C. A. P. (2020). Trajetória da privatização do sistema de saúde chileno (1924-2005). Saúde em Debate, 44, $541-555$.

Costa, H., Canto, F. L. D., \& Pinto, A. L. (2020). Google Scholar Metrics e a proposta do novo Qualis: impacto dos periódicos brasileiros de Ciência da Informação. Informação \& Sociedade: Estudos; v. 30 n. 1 (2020), 24(2).

Costa, N. D. R. (2014). Comunidade epistêmica e a formação da reforma sanitária no Brasil. Physis: Revista de Saúde Coletiva, 24, 809-829.

COSTA, Nilson do Rosário. O regime regulatório e o mercado de planos de saúde no Brasil. Ciência \& Saúde Coletiva, 13, 1453-1462.

d'Ávila, L. S., Andrade, E. I. G., \& Aith, F. M. A. (2020). A judicialização da saúde no Brasil e na Colômbia: uma discussão à luz do novo constitucionalismo latino-americano. Saúde e Sociedade, 29, e190424.

Druck, G. (2016). A terceirização na saúde pública: formas diversas de precarização do trabalho. Trabalho, educação e saúde, 14, 15-43.

Garrido-Pinzón, J., \& Bernardo, M. H. (2017). Vivências de trabalhadores da saúde em face da lógica neoliberal: um estudo da atenção básica na Colômbia e no Brasil. Cadernos de Saúde Pública, 33, e00050716.

Göttems, L. B. D., \& Mollo, M. D. L. R. (2020). Neoliberalism in Latin America: effects on health system reforms. Revista de saude publica, $54,74$.

Harvey, D. (2007). Neoliberalism as creative destruction, ANNALS. AAPSS, 610, 22-44.

Ibarra, D. (2011). O neoliberalismo na América Latina. Brazilian Journal of Political Economy, 31(2), 238-248.

Kingfisher, C., \& Maskovsky, J. (2008). Introduction: The limits of neoliberalism.

Langbecker, A., Castellanos, M. E. P., \& Catalán-Matamoros, D. (2020). Quando os sistemas públicos de saúde são notícia: uma análise comparativa da cobertura jornalista no Brasil e na Espanha. Ciência \& Saúde Coletiva, 25, 4281-4292.

Lara, L. D., Guareschi, N. M. D. F., \& Bernardes, A. G. (2016). Reforma sanitária e a privatização da saúde em um contexto biopolítico de garantia de direitos. Psicologia \& Sociedade, 28(2), 360-368.

Marques, R., Ximenes, S. B., \& Ugino, C. K. (2018). Governos Lula e Dilma em matéria de seguridade social e acesso à educação superior. Brazilian Journal of Political Economy, 38(3), 526-547.

Meireles, E. C. D., \& El-Aouar, W. A. (2010). O desafio das ONGs ante a minimização do papel do Estado no cenário global. REGE Revista de Gestão, 9(3).

Morais, H. M. M. D., Albuquerque, M. D. S. V. D., Oliveira, R. S. D., Cazuzu, A. K. I., \& Silva, N. A. F. D. (2018). Organizações Sociais da Saúde: uma expressão fenomênica da privatização da saúde no Brasil. Cadernos de Saúde Pública, 34, e00194916.

Morosini, M. V. G. C., Fonseca, A. F., \& Baptista, T. W. D. F. (2020). Previne Brasil, Agência de Desenvolvimento da Atenção Primária e Carteira de Serviços: radicalização da política de privatização da atenção básica? Cadernos de Saúde Pública, 36, e00040220.

Noronha, J. C. D., Noronha, G. S. D., Pereira, T. R., \& Costa, A. M. (2018). Notas sobre o futuro do SUS: breve exame de caminhos e descaminhos trilhados em um horizonte de incertezas e desalentos. Ciência \& Saúde Coletiva, 23, 2051-2059.

Ocké-Reis, C. O. (2012). SUS: o desafio de ser único. SciELO-Editora FIOCRUZ.

Paim, J. S., \& Teixeira, C. F. (2007). Configuração institucional e gestão do Sistema Único de Saúde: problemas e desafios. Ciência \& saúde coletiva, 12, 1819-1829.

Paiola, G. C. (2014). Os alicerces econômicos do Brasil atual: heranças de Fernando Henrique Cardoso para seus sucessores. Revista Espaço Acadêmico, 13(155), 51-60.

Pimenta, M. D. C. A, \& Pimenta, L. F. (2011). Globalização e desafios urbanos: Políticas públicas e desigualdade social nas cidades brasileiras. EURE (Santiago), 37(112), 43-61.

Saffer, D. A., Mattos, L. V., \& Rego, S. R. D. M. (2020). Nenhum Serviço de Saúde a Menos: movimentos sociais, novos sujeitos políticos e direito à saúde em tempos de crise no Rio de Janeiro, Brasil. Saúde em Debate, 44, 147-159.

Santos, M. A. (2013). Lutas sociais pela saúde pública no Brasil frente aos desafios contemporâneos. Revista Katálysis, 16(2), 233-240.

Silva, H. P., \& Caxias, M. C. L. (2016). Benefícios para alguns, prejuízos para muitos: razões e implicações da adoção da dupla porta de entrada em hospitais universitários. Saúde e Sociedade, 25, 808-820.

Sousa, A. M. D. C. (2014). Universalidade da saúde no Brasil e as contradições da sua negação como direito de todos. Revista Katálysis, 17(2), $227-234$.

Sposati, A. (2018). Descaminhos da seguridade social e desproteção social no Brasil. Ciência \& Saúde Coletiva, 23, 2315-2325.

Valla, V. V. (1998). Sobre participação popular: uma questão de perspectiva. Cadernos de Saúde Pública, 14, S07-S18. 